\title{
Adherencias de los labios menores en niñas prepuberales asintomáticas: tratar o no tratar
}

\author{
G. Orejón de Luna ${ }^{\mathrm{a}}$, M. Fernández Rodríguez ${ }^{\mathrm{b}}$ \\ aPediatra. CS General Ricardos. Madrid. España. \\ ${ }^{b}$ Pediatra. CS Potes. Madrid. España.
}

Rev Pediatr Aten Primaria. 2009; I :667-75

Gloria Orejón de Luna, gloriaglo04@hotmail.com

\begin{abstract}
Resumen
Escenario clínico: niña de 2 años que acude a la consulta para un control de salud. En la exploración se aprecia adherencia en los labios menores (sinequia vulvar). Ante este hallazgo, los padres preguntan por qué se ha producido y, sobre todo, si es necesario un tratamiento. Usted conoce el tratamiento tópico con crema de estrógenos, pero tiene dudas sobre su eficacia y sobre si está indicado si la niña se muestra asintomática.

Pregunta clínica: en niñas pequeñas con adherencias de los labios menores, ¿está indicado el tratamiento tópico con estrógenos?

Justificación: la adherencia de los labios menores es uno de los problemas ginecológicos más frecuentes en niñas prepuberales. El 1,8\% de éstas la padece en algún momento de su vida. El pico de mayor incidencia (3,3\%) se produce entre los 13 y los 23 meses de edad. Los estrógenos tópicos se han indicado como tratamiento médico inicial en la mayoría de los casos, aunque en algunas ocasiones se ha optado por la separación manual e incluso la quirúrgica, sobre todo en casos graves. Sin embargo, no están claras ni la indicación ni la eficacia de los citados tratamientos. Tampoco se conoce el pronóstico a largo plazo de esos procedimientos.

Resolución del escenario clínico: cuando los padres vuelven a la consulta, les explica que debido a que la niña no presenta síntomas, existe la posibilidad de que las adherencias se resuelvan espontáneamente con el tiempo, así como que el no tratarlas inicialmente no aumenta el riesgo de que aparezcan síntomas, por lo que no cree indicado iniciar ningún tipo de tratamiento. Acuerda con la familia hacer un seguimiento clínico y explica los síntomas que deben vigilar; asimismo, les informa también de las posibilidades terapéuticas que existen en el caso de que la niña dejara de estar asintomática.
\end{abstract}

Palabras clave: Adherencias labiales, Estrógenos tópicos.

Abstract

Clinical scenario: a two year old girl is taken to the office for a well child care control. Labial adhesions are noticed in the clinical exam. The parents ask about the reason of this condition and, more important, if it is necessary to treat it. You know the topical treatment

Este artículo se publica simultáneamente con la revista electrónica Evidencias en Pediatría (EeP, www.aepap.org/EvidPediatr/index.htm). Las autoras declaran no presentar conflictos de intereses en relación con la preparación y la publicación de este artículo. 
with estrogens, but you doubt about its effectiveness and if it is indicated if the girl is asymptomatic.

Clinical question: in small girls with labial adhesions, is the estrogen topical therapy indicated?

Justification: labial adhesions is one of the most frequent gynecologic disorders in prepubertal girls; $1.8 \%$ of them suffer from it during some time in their lives. The highest incidence (3.3\%) happens between 13 and 23 months of age. Topical estrogens have been used as the initial therapy in most of the cases, although manual separation or even surgery have been undertaken, especially in severe cases. However, the indication and effectiveness of the mentioned treatments are not clear. The long time prognosis of these procedures is not known either.

Clinical scenario resolution: when the parents come back to the office, you explain them that due to the fact that the girl doesn't have symptoms, there is the possibility that the adhesions solve spontaneously with time, and not treating them initially doesn't increase the risk of symptoms, so that you don't consider to start any kind of therapy. You agree with the family on a clinical follow up and explain them the symptoms to keep watch on; also, you inform them on the existing possibilities of therapy in case the girl stopped been asymptomatic.

Key words: Labial adhesions, Topical estrogens.

\section{Escenario clínico}

Niña de 2 años que acude a la consulta para un control de salud. Está asintomática y los padres no comentan ningún problema. En la exploración se aprecia adherencia en los labios menores (sinequia vulvar). Usted pregunta ya de forma dirigida sobre posibles síntomas y los padres insisten en que no han notado nada a la niña. Ante este hallazgo, los progenitores preguntan por qué se ha producido y, sobre todo, si es necesario un tratamiento. Usted conoce el tratamiento tópico con crema de estrógenos, pero tiene dudas sobre su eficacia, así como si está indicado si la niña está asintomática. Decide informarse sobre el tema y cita a los padres en unos días para darles una solución.

\section{Pregunta clínica}

En niñas pequeñas con adherencias de los labios menores, ¿está indicado el tratamiento tópico con estrógenos?

\section{Búsqueda bibliográfica}

1. Biblioteca Cochrane Plus. Estrategia de búsqueda: con distintos términos descriptores: "labial adhesions", "labial fusion", "minora labial adhesion", "synechia vulvae". Búsqueda en bases de datos de revisiones sistemáticas y en la base de datos de ensayos clínicos (CENTRAL). No se recuperó ningún estudio relacionado con la pregunta clínica. 
2. TRIP Database. Estrategia de búsqueda: términos descriptores: "labial adhesions" and "girls" or "pediatrics", en título y texto de los documentos. Estudios recuperados: 22. Estudios de interés para responder a la pregunta clínica: tres.

3. PubMed. Estrategia de búsqueda: términos descriptores: "labial adhesions"AND ((clinical[Title/Abstract] AND trial[Title/Abstract]) OR clinical trials [MeSH Terms] OR clinical trial[Publication Type] OR random*[Title/Abstract] OR random allocation[MeSH Terms] OR therapeutic use[MeSH Subheading]) Limits: added to PubMed in the last 10 years, published in the last 10 years, Humans, All Child: 0-18 years. Estudios recuperados: ocho. Estudios de interés para responder a la pregunta: tres.

Las búsquedas efectuadas en TRIP Database y PubMed recuperaron los mismos artículos ${ }^{1-3}$ :

1. Muram D. Treatment of prepuberal girls with labial adhesions. J Pediatr Adolesc Gynecol. 1999;12:67-70.

2. Schober J, Dulabon L, Martín-Alguacil N, Kow LM, Pfaff D. Significance of topical estrogens to labial fusion and vaginal introital integrity. J Pediact Adolesc Gynecol. 2006;19:337-9.

3. Sover T. Topical estrogen therapy in labial adhesions in children: thera- peutic or prophylactic? J Pediatr Adolesc Gynecol. 2007;20:241-4.

\section{Resumen estructurado de los artículos seleccionados}

Muram D. Treatment of prepuberal girls with labial adhesions. J Pediatr Adolesc Gynecol. 1999;12:67-70

Objetivo: valorar la eficacia de los estrógenos tópicos en el tratamiento de las adherencias labiales en niñas prepuberales.

Diseño: estudio descriptivo retrospectivo.

Emplazamiento: clínica ginecológica para niñas y adolescentes de Memphis (EE. UU.).

Población de estudio: se incluyeron 262 niñas de un total de 289 de 1 a 17 años, remitidas a la clínica para tratamiento con el diagnóstico de adherencias labiales desde octubre de 1982 hasta junio de 1996. Se excluyó a las niñas que estaban asintomáticas al inicio y permanecieron sin síntomas a lo largo del estudio.

Intervención: en tres niñas con adherencias y fusión densa y fibrosa de los labios menores y con retención urinaria se realizó la separación quirúrgica. En las 259 niñas restantes se aplicó un tratamiento tópico con estrógenos (crema 
vaginal 0,625 mg de estrógenos conjugados) durante 10-14 días. De ellas, 138 precisaron además separación manual bajo anestesia tópica, y en 26 niñas de este último grupo hubo que realizar la separación quirúrgica bajo anestesia general. Durante los 5 días posteriores a la intervención se indicó un tratamiento tópico con estrógenos para evitar recurrencias.

Medición del resultado: se describen durante el seguimiento la tasa de curación en los distintos escalones terapéuticos, así como la tasa de recurrencia con cada uno de los tratamientos aplicados.

Resultados principales: el 46,7\% (IC 95\%: 40,4 a 52,9) (121/259) de las niñas se curó con el tratamiento tópico, con un porcentaje de recurrencias del $11,5 \%$ (IC $95 \%: 5,5$ a 17,7) (14/121). En 112 de las 138 niñas que se sometieron a la separación manual (81\%; IC 95\%: 74,3 a 88) se constató curación; las adherencias reaparecieron en 18 (16\%). De los 26 casos en los que se precisó separación quirúrgi$\mathrm{ca}$, en cuatro (15,3\%; IC 95\%: 4,3 a $34,8)$ las adherencias volvieron a aparecer. Las niñas que precisaron separación quirúrgica tenían 3 o más años de edad y sus adherencias labiales eran muy fibrosas y de larga evolución.

Conclusiones de los autores: es posible que muchas pacientes con adhe- rencias labiales precisen tratamientos adicionales a los estrógenos tópicos. Niñas con adherencias labiales densas y fibrosas, en las que previamente se ha realizado una separación quirúrgica, responden peor a dicho tratamiento médico.

Conflictos de intereses: no constan.

Fuente de financiación: no consta.

Schober J, Dulabon L, Martín-Alguacil $N$, Kow LM, Pfaff D. Significance of topical estrogens to labial fusion and vaginal introital integrity. J Pediact Adolesc Gynecol. 2006;19:337-9.

Objetivo: valorar la eficacia y seguridad de los estrógenos tópicos en el tratamiento de las adherencias de los labios menores.

Diseño: estudio descriptivo retrospectivo.

Emplazamiento: centro médico universitario de Erie (EE. UU.).

Población de estudio: 109 niñas de entre 3 meses y 10 años diagnosticadas de adherencias de los labios menores.

Intervención: se revisaron retrospectivamente las historias clínicas de las niñas seleccionadas que recibieron tratamiento tópico con estrógenos, así como de las niñas que precisaron tratamiento quirúrgico por tener adherencias labia- 
les muy gruesas y refractarias al tratamiento médico.

Medición de los resultados: las variables medidas fueron: duración del tratamiento, tasa de curación, tasa de recurrencia, tiempo transcurrido desde el tratamiento hasta la recurrencia, porcentaje de niñas que precisaron tratamiento quirúrgico y efectos adversos.

Resultados principales: la duración del tratamiento fue de 3,7 meses (rango: 1-36 meses), con una tasa de curación del 79\% (IC 95\%: 71,3 a 87,5) (85/107). En el 41\% (IC 95\%: 31,3 a $50,9)$ de los casos $(44 / 107)$ hubo recurrencia de los síntomas al menos en una ocasión, y en algunos casos hasta en cinco, en un período de tiempo de entre 2 y 72 meses tras el tratamiento. Una niña no precisó ningún tipo de tratamiento por resolución espontánea de las adherencias antes del inicio del tratamiento. El tratamiento quirúrgico se indicó en 22 de las 107 niñas (21\%; IC $95 \%$ : 12,4 a 28,7 ), con buenos resultados en 19 casos. La tasa de recurrencia posquirúrgica fue del 10\% (IC 95\%: 1,2 a 30,4$)(2 / 21)$ y fue necesario realizar una segunda separación quirúrgica de las adherencias por la falta de respuesta al tratamiento tópico con estrógenos. Los efectos adversos declarados con el tratamiento tópico fueron: un mínimo desarrollo mamario en 6 niñas y sangrado vaginal en otra.

Conclusión de los autores: los estrógenos tópicos son eficaces en el tratamiento de las adherencias de los labios menores.

Conflictos de intereses: no constan.

Fuente de financiación: no consta.

Soyer T. Topical estrogen therapy in labial adhesions in children: therapeutic or prophylactic? J Pediatr Adolesc Gynecol. 2007;20:241-4.

Objetivo: valorar la eficacia de los estrógenos tópicos como tratamiento o como profilaxis de las adherencias labiales en niñas.

Diseño: estudio de cohortes retrospectivo.

Emplazamiento: unidad de cirugía pediátrica de un hospital de Ankara (Turquía).

Población de estudio: 49 niñas diagnosticadas de adherencias de los labios menores, que se distribuyeron en 3 cohortes de estudio: grupo de tratamiento conservador (18 niñas), con una media de edad de 14,05 $( \pm 4,19)$ meses que estaban asintomáticas y no habían recibido ningún tratamiento previo; grupo de separación manual (14 niñas), con una media de edad de 14,64 $( \pm 4,60)$ 
meses, con síntomas, y en las que el tratamiento médico no había sido eficaz; y grupo de separación manual con profilaxis (17 niñas), con una media de edad de 12,82 $( \pm 4,34)$ meses, con síntomas, y que no habían recibido previamente ningún tratamiento.

Intervención: en el primer grupo se realizó un tratamiento conservador y se indicaron estrógenos tópicos (crema vaginal 0,625 mg de estrógenos conjugados) dos veces al día durante 14 días. En el segundo grupo se realizó una separación manual bajo anestesia tópica (lidocaína 5\%). En el tercer grupo, además de la separación manual, realizada de forma similar, se administró, durante los 5 días posteriores a la intervención, crema de estrógenos tópicos dos veces al día como profilaxis para evitar recurrencias. Se realizó un seguimiento hasta los 9 meses después del tratamiento (las visitas tuvieron lugar a los 3 y a los 9 meses).

Medición del resultado: las principales variables valoradas fueron: tasa de curación a lo largo del seguimiento y porcentaje de recurrencias.

Resultados principales: el 66,6\% (IC $95 \%$ : 40,9 a 86,6) $(12 / 18)$ de las niñas del grupo de tratamiento tópico con estrógenos se había curado al tercer mes del tratamiento y el $55,5 \%$ (IC
95\%: 22,3 a 68,5$)(10 / 22)$, en el noveno mes. El porcentaje de recurrencias fue del 11\% (IC 95\%: 1,1 a 29,1) $(2 / 22)$. En el segundo grupo, la curación se produjo en el $85 \%$ (IC 95\%: $28,8$ a 82,3$)(8 / 14)$ al tercer y noveno mes de seguimiento y hubo recurrencias en dos pacientes (14,2\%; IC 95\%: 1,7 a 42,8). En todas las niñas incluidas en el grupo de separación manual y profilaxis con estrógenos tópicos (17/17; 100\%; IC 95\%: 80,4 a 100), se obtuvo curación y no hubo recurrencias durante todo el seguimiento. Los síntomas que referían las pacientes incluidas en el segundo y tercer grupo fueron: disuria, infecciones vulvares recurrentes e infecciones urinarias. Los ensayos clínicos aleatorizados (ECA) descritos fueron pigmentación vulvar en 4 pacientes ( $22 \%$ ).

Conclusión de los autores: en las pacientes con adherencias labiales y síntomas secundarios está indicada la separación manual. En las pacientes asintomáticas, el tratamiento tópico con estrógenos es adecuado, aunque su eficacia es limitada. El tratamiento tópico con estrógenos es eficaz de forma profiláctica tras la separación manual para prevenir recurrencias.

Conflictos de intereses: no constan.

Fuente de financiación: no consta. 


\section{Comentario crítico}

Justificación: la adherencia de los labios menores es uno de los problemas ginecológicos más frecuentes en niñas prepuberales. El 1,8\% de éstas lo padece en algún momento de su vida. El pico de mayor incidencia $(3,3 \%)$ se produce entre los 13 y los 23 meses de edad ${ }^{4}$. Aunque la causa se desconoce, se ha relacionado con el bajo nivel de estrógenos que existe en las niñas antes de la pubertad. La clínica es muy variable. Las niñas pueden estar asintomáticas o tener síntomas leves (como irritaciones vaginales), síntomas más importantes (como infecciones vaginales y/o urinarias de repetición) o más graves (como incontinencia y retención urinaria). Los estrógenos tópicos se han indicado como tratamiento médico inicial en la mayoría de los casos, aunque en algunas ocasiones se ha optado por la separación manual e incluso quirúrgica, sobre todo en casos graves. Sin embargo, no están claras ni la indicación ni la eficacia de los citados tratamientos. Tampoco se conoce el pronóstico a largo plazo de esos procedimientos.

Validez o rigor científico: los estudios tienen las limitaciones propias de los estudios retrospectivos y no experimentales. Sin embargo, el diseño -principalmente de los estudios de Muram y
Soyer- parece adecuado para valorar la práctica clínica real a falta de ECA. El tamaño de la muestra es grande, teniendo en cuenta otros estudios, y por las características descritas de las muestras de población, éstas parecen representativas. Sólo hubo dos casos que se perdieron en el estudio de Schober. Las cohortes comparadas en los distintos estudios no son exactamente iguales. Muram excluye del estudio a las niñas que fueron asintomáticas. Sin embargo, en los otros dos estudios éstas si se incluyeron, por lo que es difícil concluir una indicación clara en este grupo de población. Las intervenciones sí fueron similares en los tres estudios, por lo que los resultados del efecto de éstas en los diferentes grupos de estudio parecen válidos. Los tiempos de seguimiento también fueron variables, lo que ha podido influir en la diferencia en la tasa de recurrencia. En el estudio de Muram no se hace referencia a los efectos adversos del tratamiento y en ninguno se habla de los efectos a largo plazo.

Importancia clínica: en los estudios revisados, la tasa de curación en las adherencias de los labios menores tras el tratamiento con estrógenos tópicos fue del $46,7 \%, 66,6 \%$ y $79 \%$, respectivamente. Los resultados fueron peores en las niñas mayores de 3 años y en los ca- 
sos de adherencias densas y fibrosas. La tasa de recurrencia fue del $11 \%$ en dos de los estudios y del $41 \%$ en el de Schober, en el que el tiempo de seguimiento fue más largo. Resultados similares se obtienen en otros estudios de similares características ${ }^{5,6}$. Los efectos adversos descritos (desarrollo mamario, hiperpigmentación vulvar), aunque no son frecuentes y desaparecen al suspender la medicación, sí pueden llegar a ser importantes, por la angustia que generan en la familia. Para evitarlo, se ha propuesto el tratamiento con betametasona al 0,05\%, usado ampliamente en el tratamiento de la fimosis en niños. A este respecto, en un estudio también retrospectivo ${ }^{7}$, Myers concluye que dicho tratamiento es eficaz, pero la muestra estaba constituida por 19 niñas, 14 de las cuales habían sido tratadas previamente con estrógenos tópicos y una de ellas, con tratamiento quirúrgico.

Aplicabilidad a la práctica clínica: el tratamiento tópico con estrógenos en las adherencias de los labios menores es eficaz y seguro, con escasos y poco importantes efectos adversos, por lo que se puede considerar de primera elección, principalmente en las niñas con síntomas secundarios. En los casos refractarios al tratamiento médico se podría plantear la separación manual y, si ésta no es eficaz, la separación quirúrgica. Lo que también parece claro es que para evitar o disminuir recurrencias posteriores a dichos tratamientos, se deben administrar estrógenos tópicos durante cinco días después de la manipulación. A falta de ECA y de estudios experimentales correctamente diseñados, no se puede establecer una indicación clara en el tratamiento de niñas asintomáticas. Es más, dada la tasa de recurrencias del tratamiento médico, que podría dar lugar a la indicación de tratamientos menos conservadores en niñas sanas, el hecho de que no tratar las adherencias labiales en niñas sin síntomas no conlleva un mayor riesgo de que las desarrollen posteriormente, así como que las adherencias se pueden resolver espontáneamente a medida que las niñas crecen, hace pensar que en este grupo estaría más indicado un manejo sólo con seguimiento clínico, como opinan otros autores ${ }^{8}$.

\section{Resolución del escenario clínico}

Cuando los padres vuelven a la consulta les explica que, puesto que la niña no presenta síntomas, existe la posibilidad de que las adherencias se resuelvan espontáneamente con el tiempo, así como que el no tratarlas inicialmente no aumenta el riesgo de que aparezcan sín- 
tomas; por ello, señala que no cree indicado iniciar ningún tipo de tratamiento. Acuerda con la familia hacer un seguimiento clínico y explica los síntomas que deben vigilar. Les informa también de las posibilidades terapéuticas que existen en el caso de que la niña dejara de estar asintomática.

\section{Bibliografía}

1. Muram D. Treatment of prepuberal girls with labial adhesions. J Pediatr Adolesc Gynecol. 1999;12:67-70.

2. Schober J, Dulabon L, Martín-Alguacil N, Kow LM, Pfaff D. Significance of topical estrogens to labial fusion and vaginal introital integrity. J Pediact Adolesc Gynecol. 2006;19:337-9.

3. Sover T. Topical estrogen therapy in labial adhesions in children: therapeutic or prophylactic? J Pediatr Adolesc Gynecol. 2007;20:241-4.

4. Leung AK, Robson WL, Tay-Uyboco J. The incidence of labial fusion in children. J Paediatr Child Health. 1993;29:235.
5. Leung AK, Robson WL, Kao CP, Liu EK, Fong $\mathrm{JH}$. Treatment of labial fusion with topical estrogen therapy. Clin Pediatr (Phila). 2005;44 (3):245-7.

6. Bacon JL. Prepuberal labial adhesions: evaluation of a referral population. Am J Obstet Gynecol. 2002;187(2):327-31.

7. Myers JB, Sorensen CM, Wisner BP, Furness $P D$, Passamaneck $M$, Koyle MA. Betamethasone cream for the treatment of pre-pubertal labial adhesions. J Pediatr Adolesc Gynecol. 2006;19: 407-11.

8. Omar HA. Opinion in pediatric and adolescent gynecology. J Pediatr Adolesc Gynecol. 2000;13:183-6. 